\title{
An Extracting Method of 2D Property Line for House Property
}

\author{
Yunfei Shi* and Lingling Zhang \\ School of Resource and Environment, Linyi University, 276000 Linyi, China \\ ${ }^{*}$ Corresponding author
}

\begin{abstract}
D property line is a combined boundary line of geometry and semantics, extraction of which is basis for reconstruction of right volumes (house property). Research on extraction of property boundaries for flat-layer right volumes in the slab-type apartment buildings has been tried in this article. Besides, four rules for boundary extraction of slab-type right volumes have been proposed. Floor plans expressed in single line has been acquired through extraction of the central line of walls and side boundary of window and balcony. Furthermore, "welding" has been conducted on edges extracted. Area ratio method has been raised so as to divide each property right entity in the plans, and the central line extracted with this method will divide floor plans into multiple units. On this basis, boundary search algorithm based on the maximum angel has been proposed, which can be used to trace boundary of each right volumes.
\end{abstract}

Keywords-right volumes; floor plans; $3 D$ cadastre; real estate management

\section{INTRODUCTION}

In recently years, many countries have began to develop 3D cadastral management mode(Stoter 2005; Guo,2010; Guo,2012) on the basis of research on 3D geographic information system, trying to solve the problems which cannot comprehensively reflect land use conditions in the existing land registration and cadastral management system with 3D cadastral management mode so as to carry out registration and recording on use status of certain particular land to accurately reflect reasonable application of 3D land space. Right volumes (Stoter,2004) refers to registration object of 3D cadastre, which is the basis unit to carry out 3D cadastral registration, inquiry, analysis, calculation and other operations. Registration in the form of right volumes can accurately record spatial property range of property units and provide effective methods to determine spatial property. However, the quantity of right volumes in the city is huge. How to rapidly and economically accomplish reconstruction of right volumes is the key for precision management of 3D property rights. However, in actual world, majority of right volumes are regular and provided with corresponding 2D graphic data (floor plans) and height information. Therefore, the method of "stretching" 2D graphics can be employed to generate right volumes. Since the reconstruction of right volumes is intended for determination of property range and boundary of property units in 3D space, it only requires constructing boundary geometry of the right volumes when the reconstruction is carried out. Besides, it is unnecessary to carry out reconstruction on the interior pattern, function division, etc., which signifies that, during reconstruction of the right volumes, it only requires recognizing 2D-property line of various right volumes in the floor plan. 2D-property line is boundary line geometry and semantic of which are composite. Besides, it is not quite feasible to automatically recognize this line simply with geometric algorithm. Of course, the method most commonly used is to conduct handmade delineation with mapping software (including CAD), but it will cost plenty of time and labor, and the efficiency is low. Therefore, exploration of automatic boundary-extracting method under manual intervention is provided with extensive application prospect in property-right precision management, 3D cadastre, digital city and other fields.

\section{FOUNDATION OF RESEARCH}

In this program, the project team tries to carry out semi-automatic extracting research on the property range of flat-layer right volumes. Characteristics of such right volumes are unit-style splice and a slab-type building will be established through splicing several units. Right volumes of slab-type building conforms to the following four rules:

Rule 1: As is shown in Figure I, all the apartments (units) on one floor are composed in four ways including stand-alone type, loose line mode, symmetric type and interlaced type in a slab-type building.

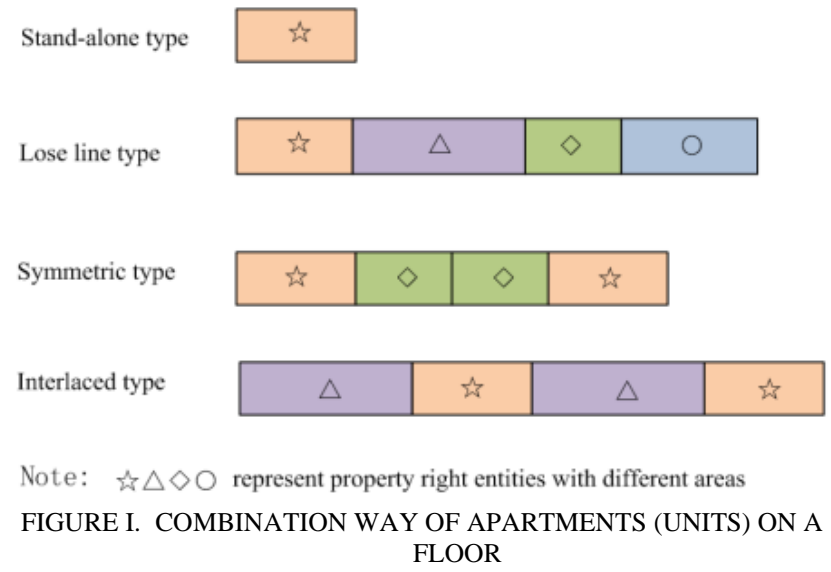

Rule 2: As for a floor in a slab-type building, regardless of the periphery that is composed of walls, doors, windows or balconies, its 2D-property line is always a continuum boundary, which signifies that we will return to the same node after having conducted rotary movement for a circle, as is shown in Figure II. 


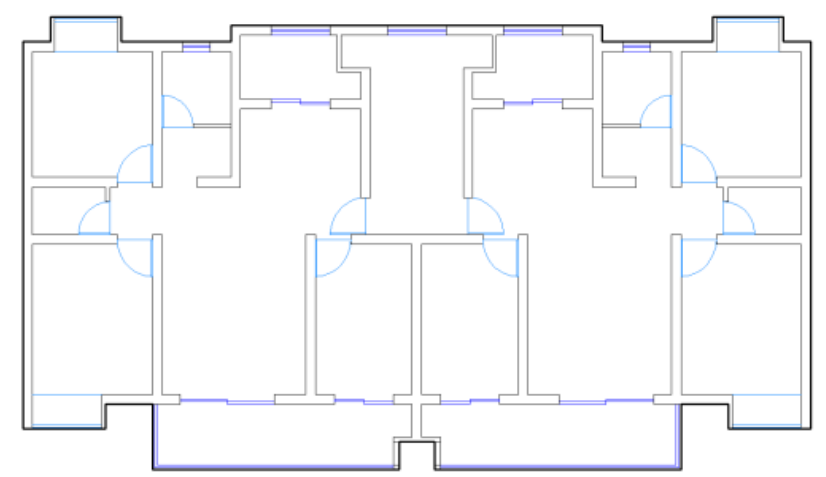

FIGURE II. OUT- ILLUSTRATION OF A FLOOR IN A SLAB-TYPE BUILDING

Rule3: Overwhelming majority of nodes are connected with the edges are in four types of "Type -”, "Type L”, "Type $\perp$ " and "Type + ". Seldom can connection of “ $*$ type" be seen.

Rule 4: Ends of walls can be observed at both edges of the window, but balconies are not drawn on the end of the walls.

\section{Automatic EXtraction OF 2D-Property Line}

2D property boundaries of stab-type right volumes shall be extracted with the following method and in accordance with the rules stated above:

\section{A. Extraction of Drawing Layer}

Data pre-processing including data check, topological correction shall be carried out for floor plans and data sources shall be purified. Besides, characters irrelevant to reconstruction and drawing layers of stairs and other structures shall be filtered out while walls, doors, windows and balcony drawing layers relevant to the boundary shall be reserved.

\section{B. Extraction of Central Line of Walls}

Since all the area of compartment walls between apartments, divisions between the apartment and communal building space, outer walls (including gables) and other communal walls are reckoned in the wall area in accordance with half of the horizontal projected area, central lines of the walls shall be extracted, method of which shall be conforms to the algorithm from(Yang,2011).

The algorithm shall be divided into two steps: (1) Central-line positions shall be calculated with the formula and then the central lines shall be extracted; (2) Mutual divisions shall be conducted for the central lines extracted, and repetitions may come up. Subsequently, unnecessary central lines shall be removed on the basis of (1)and the neighboring central lines shall be connected with established threshold value, as is illustrated in Figure III.

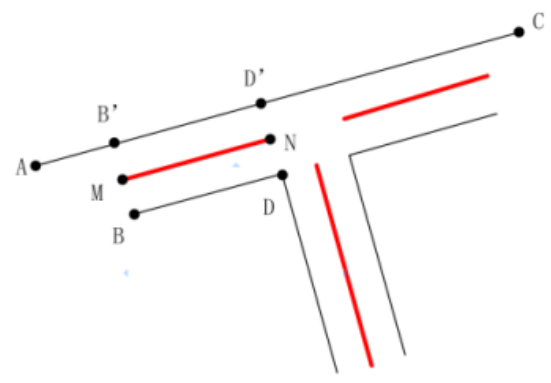

\section{FIGURE III. EXTRACTION OF CENTRAL LINES OF WALLS}

The extraction formulas for the corresponding central lines in Figure III are as follows:

$$
\begin{aligned}
& \left.B^{\prime}=\frac{\overrightarrow{A C}}{|\overrightarrow{A C}|}|\overrightarrow{A B}| \times \frac{\overrightarrow{A B} \bullet \overrightarrow{A C}}{|\overrightarrow{A B}||\overrightarrow{A C}|}\right)+A \\
& D^{\prime}=\frac{\overrightarrow{A C}}{|\overrightarrow{A C}|}\left(|\overrightarrow{A D}| \times \frac{\overrightarrow{A D} \bullet \overrightarrow{A C}}{|\overrightarrow{A D}||\overrightarrow{A C}|}\right)+A \\
& M=\frac{B+B^{\prime}}{2}, M=\frac{D+D^{\prime}}{2}
\end{aligned}
$$

Here, $\mathrm{M}, \mathrm{N}$ refers to coordinate pair of such as coordinates $(\mathrm{x}, \mathrm{y})$. Extraction of central lines for four-type walls has been demonstrated in Figure IV. Schematic diagram of central lines having been connected is shown in the graphic on the second line in Figure IV.

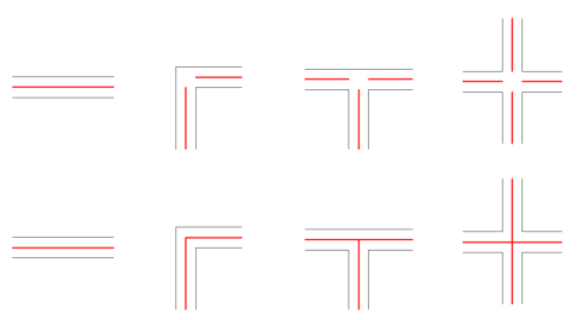

FIGURE IV. EXTRACTION OF FOUR-TYPE CENTRAL LINES OF WALLS

\section{Recognition and Extraction of Edge Lines of Windows and Balconies}

Geometric expressions of windows are relatively simple compared to those of walls, all of which are expressed with 3 to 4 parallel lines with similar distances. Balconies can be considered as windows with right angles, which can also be expressed with parallel lines. Segments used to express windows are provided with two characteristics: at least three paralleled segments are required; all of the segments shall be mutually paralleled and perpendicular distance between segments shall be no more than WallWideMax. Based on these two characteristics, buffer circle idea shall be introduced during recognition of windows, that is, a circle shall be produced by taking the segments expressing windows as center of the circle. In case that the existing segments are inner-side lines such as two inner-side fine lines as is shown in Figure V, buffer circle produced by taking WallWideMax as the semi-diameter is the circle shown on the right side of 
Figure V. In case that the existing segments are outer-side lines, the buffer circle produced is the circle shown on the left side of Figure $\mathrm{V}$, and the segment with the farthest distance from the existing segment may be come into contact with this circle. Therefore, semi-diameter of circles shall be determined slightly larger than that of the maximum width value of the walls and thus WallWideMax +1 shall be adopted. It can be maintained in such way that endpoints affiliated to the same end of the same window can totally fall into the buffer circle. In case that endpoints falling into the buffer circle is more than three (including the existing one), it can be indicated that these segments may be the corresponding ones of the same window, and judgments with other conditions shall be continuously carried out. Otherwise, directly jump to the next segment.

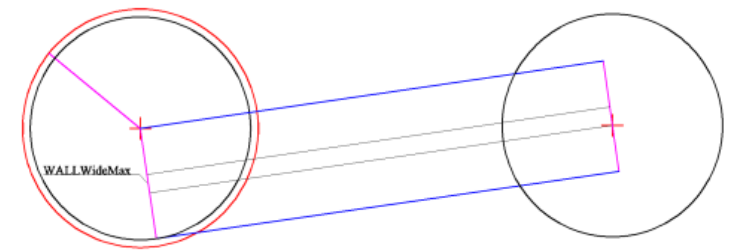

FIGURE V. RELATION OF WINDOW LINES AND BUFFER CIRCLE

\section{Search on Boundary of Right Volumes based on Area Ratio Method}

Based on Rule 1 stated above, "all the apartments (units) on a floor in a slab-type building are composed in four types including stand-alone type, loose line mode, symmetric expression and interlaced type." It means that spatial distribution of right volumes in a slab-type building is provided with certain regularity: widths of all the right volumes on the entire slab-type building are identical, and the right volumes on each floor are composed of one of the four types, which can be expressed as $120 \mathrm{~m}^{2}+120 \mathrm{~m}^{2}$ or 120 $\mathrm{m}^{2}+140 \mathrm{~m}^{2}+140 \mathrm{~m}^{2}+120 \mathrm{~m}^{2}$, etc. Length and width of one floor of the building are both determined. As for different house types, in case that the widths are equal, length of unit reflects its area. Conversely, in case that the area is provided, length of the unit can be calculated and thus boundary lines among units can be found. First, the length for a floor of building can be obtained according to the law and then $L$ shall be divided by taking house-type area of the entire building as the constraint condition. For example, as for a floor of building with $\mathrm{n}$ units of $A_{1}+A_{2}+\ldots+A_{n}$ ( $\mathrm{A}_{\mathrm{n}}$ is used to indicate area), the following formula is adopted for calculation:

$$
l_{i}=\frac{A_{i}}{A_{1}+A_{2}+\ldots+A_{n}} \times L
$$

$l_{i}$ refers to the length Unit $i$ has occupied in the entire floor of the building.

\section{E. Boundary Search Based on Maximum Angle}

When central lines between units are found, floor plan shall be divided into multiple units with central lines. Since certain edge lines have been cut off due to division of central lines, pendent edges will be hereby formed. As is shown in Figure VI, the central line $\mathrm{BC}$ has been divided into two parts, and Figure VI is its left half. After division, edge DA becomes pendent edge. The further task is to search the external boundary of DA as the property boundary, method of which is the boundary search algorithm based on the maximum angle.

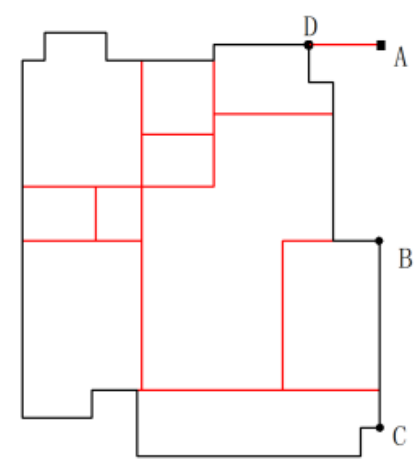

\section{FIGURE VI. ILLUSTRATION OF CENTRAL-LINE DIVISION}

Since overwhelming majority of nodes and edges are connected in the four types of “Type - ", “Type L ”, “Тype

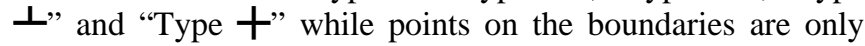
provided with the first three connecting modes. The following algorithm has been designed according to Rule 3.Point LowLeftVertex at the leftmost position shall be searched; Search on the edge related to LowLeftVertex shall be carried out, which num shall be recorded and reserved in Vec_Edge. Since there may be pendent edges in the edges related to LowLeftVertex, search and elimination shall be carried out. Since LowLeftVertex are angle points of the unit, num of which can only be 2, that's, there are only two related edges and one angle. No problem of maximum angle exists. Comparison on non-communal points of two related edges shall be carried out, the edge where the endpoint with smaller vertical coordinate is located shall be taken as the starting edge, and thus the other edge will be the one we are searching. Search on the following targeted edge shall be carried out on the basis of the other endpoint of the targeted edge; In case that num=2, directly jump to the next related edge to search; Otherwise, search on the related edges of the existing endpoint (it is assumed as point $\mathrm{O}$ in Figure VII) shall be carried out. In case that the related edges are pendant edges, they shall be eliminated. In consideration of universality of the algorithm, there shall be no orthogonality among the edges, and four edges are related with Point $\mathrm{O}$, that's, $\mathrm{OA}, \mathrm{OB}$, OC and OD. Here, D is a pendant point, and OD is pendant edge. Although included angle formed between $\mathrm{OD}$ and the anti-clockwise direction of $\mathrm{OA}$ is the maximum, OD shall be eliminated either. Included angle between other edges and the anti-clockwise direction of existing edge shall be calculated and the size shall be compared. Search shall be continuously carried out by taking the edge with the maximum angle. Termination condition: return to Point LowLeftVertex.

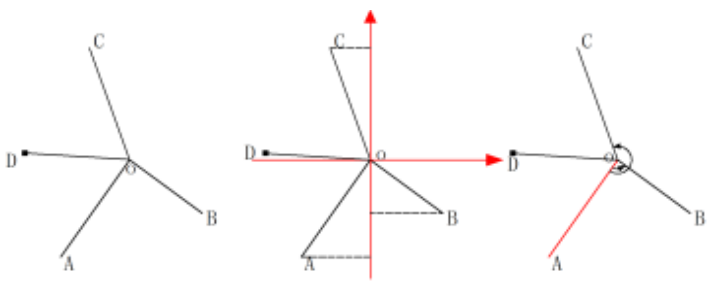

FIGURE VII. BOUNDARY SEARCH BASED ON MAXIMUM ANGLE 


\section{CONCLUSION}

Buffer circle, area ratio method, maximum angle and other technologies have been employed to recognize and extract 2D-property line for property right entities in this article, which is of certain effectiveness for slab-type property right entities. However, this algorithm can neither be used universally nor be applicable to complicated property right entities of tower-type nor circle constructions. Since semantic problem has been involved in extraction of property line, only through design of more intelligent algorithm can property boundaries of property right entities for complicated structures be extracted, which is the further research work of project team.

\section{ACKNOWLEDGEMENTS}

This work was financially supported by the National Natural Science Foundation of China (Grant No. 41201407).

\section{REFERENCES}

[1] Guo, R.Z., Ying,S.,2010.Three-dimension cadastre analysis and data delivery. China Land Science, 24(12), pp.45-51.

[2] Guo, R.Z., Ying, S., 2012. Automatic construction of 3D valid solids for 3D cadastral objects based on facet sets. Acta Geodaetica Et Cartographica Sinica, 41(4), pp.620-626.

[3] Stoter J.E.,Van Oosterom P.,2005.Technological Aspects of a 3D Cadastral Registration. International Journal of Geographical Information Science,19(6),pp.669-696.

[4] Stoter, J. E. ,2004. 3d cadastre. Delft: Delft University of Technology the Netherlands.

[5] Yang, Y., Jiang, X. T., Kuang, Y. H., 2011. Arithmetic of 3-d house reconstruction based on graphics understanding. Information \& Electronic Engineering, 09(1), p p.105-108. 